\title{
HAUSDORFF DIMENSION, ORTHOGONAL PROJECTIONS AND INTERSECTIONS WITH PLANES
}

\author{
PERTTI MATTILA
}

\section{Introduction}

Let $E$ be a plane set which is measurable with respect to the $s$-dimensional Hausdorff measure $\mathscr{H}^{s}$ with $0<\mathscr{H}^{s}(E)<\infty$. In [10] Marstrand proved among other things the following three theorems:

(1) If $1<s \leq 2$, then the Lebesgue measure $\mathscr{L}^{1}[p(E)]$ is positive for almost all orthogonal projections $p: R^{2} \rightarrow R^{1}$.

(2) If $0 \leq s \leq 1$, then the Hausdorff dimension of $p(E)$ is equal to $s$ for almost all orthogonal projections $p: R^{2} \rightarrow R^{1}$.

(3) If $1<s \leq 2$, then at $\mathscr{H}^{s}$ almost all points $x \in E$ the following is true: For almost all straight lines $l$ through $x, \mathscr{H}^{s-1}(E \cap l)<\infty$ and the Hausdorff dimension of $E \cap l$ is equal $s-1$.

Results similar to (1) and (2) were also obtained by Kaufman [7] with potential-theoretic methods. Kaufman was also able to show that the exceptional set of projections in (2) has $\mathscr{H}^{s}$ measure zero.

In this paper we generalize the results $(1)-(3)$ by replacing the dimensions 2 and 1 by arbitrary dimensions $n$ and $m, 0<m<n$. We use the method of Marstrand in (1) and (3) and that of Kaufman in (2). Certainly Marstrand's ideas could also be used to generalize (2), but Kaufman's method is shorter and gives a result on capacities and on the Hausdorff measures of the exceptional set. On the other hand, Marstrand's method allows us to improve (1) in two respects. Firstly, the conclusion $\mathscr{L}^{m}[p(E)]>0$ can be replaced by the stronger conclusion $\int N(p \mid E, y) d \mathscr{L}^{m} y=\infty$, where $N(p \mid E, y)$ is the number of points in the set $E \cap \mathrm{p}^{-1}\{y\}$. Secondly, the hypothesis that the Hausdorff dimension of $E$ is greater than $m$ can be replaced by the weaker hypothesis $C_{m}(E)>0$, where $C_{m}$ is the capacity corresponding to the Riesz kernel $|x-y|^{-m}$. As a corollary it follows that if the integral-geometric measure $\mathscr{I}_{1}^{m}(E)$ is finite, then $C_{m}(E)=0$. 
When we consider a set $E$ with $\mathscr{H}^{s}(E)>0$, two assumptions will often be equally convenient; namely, that $E$ is a Suslin set or that $E$ is $\mathscr{H}^{s}$ measurable with $\mathscr{H}^{s}(E)<\infty$, because in both cases $E$ contains a compact set $K$ with $0<\mathscr{H}^{s}(K)<\infty$. The case where $E$ is $\mathscr{H}^{s}$ measurable and $\mathscr{H}^{s}(E)=\infty$ is entirely different. In fact, assuming the continuum hypothesis Besicovitch [1] has constructed an $\mathscr{H}^{1}$ measurable plane set $G$ such that $\mathscr{L}^{2}(G)=1$ and that the only subsets of $G$ of zero $\mathscr{L}^{2}$ measure are countable sets.

Several instructive examples and remarks related to the present subject can be found in [10].

Most of the terminology and notation will be as in [5].

\section{The measures $\vartheta_{n, m}^{*}$ and $\gamma_{n, m}$}

Let $O(n)$ be the orthogonal group of the Euclidean $n$-space $R^{n}$ and let $\vartheta_{n}$ be the unique Haar measure on $O(n)$ such that $\vartheta_{n}[O(n)]=1$. If $m$ is an integer such that $0 \leq m \leq n$, then $O^{*}(n, m)$ is the space of all orthogonal projections from $R^{n}$ onto $R^{m}$, and $G(n, m)$ is the space of all $m$-dimensional linear subspaces of $R^{n}$. We shall use the $O(n)$ invariant measures $\vartheta_{n, m}^{*}$ on $O^{*}(n, m)$ and $\gamma_{n, m}$ on $G(n, m)$ (see $[5,2.7 .16(6)]$ ). If $p \in O^{*}(n, m)$ and $V \in G(n, m), A \subset O^{*}(n, m)$ and $B \subset G(n, m)$, then by definition

$$
\begin{aligned}
& \vartheta_{n, m}^{*}(A)=\vartheta_{n}\{g \in O(n): p \circ g \in A\}, \\
& \gamma_{n, m}(B)=\vartheta_{n}\{g \in O(n): g(V) \in B\} .
\end{aligned}
$$

The measures $\vartheta_{n, m}^{*}$ and $\gamma_{n, n-m}$ are related by

$$
\gamma_{n, n-m}(A)=\vartheta_{n, m}^{*}\left\{p \in O^{*}(n, m): \operatorname{ker} p \in A\right\}
$$

for $A \subset G(n, n-m)$.

If $0 \leq s \leq n$, we let $\mathscr{H}^{s}$ be the $s$-dimensional Hausdorff measure over $R^{n}$ as defined in $[5,2.10 .2(1)]$. Then $\mathscr{H}^{n}=\mathscr{L}^{n}$, the Lebesgue measure in $R^{n}$. We denote by $S^{n-1}$ the unit sphere $\left\{x \in R^{n}:|x|=1\right\}$ and, when $a \in R^{n}$ and $r>0$, by $B^{n}(a, r)$ or $B(a, r)$ the closed ball $\left\{x \in R^{n}\right.$ : $|x-a| \leq r\}$.

Throughout the rest of the paper $m$ and $n$ will be integers such that $0<m<n$.

2.4. L e m ma. There is a constant $c_{1}$ depending only on $n$ and $m$ such that

$$
\mathscr{H}^{n-1}\left\{x \in S^{n-1}:|p(x)| \leq \delta\right\} \leq c_{1} \delta^{m}
$$

for all $p \in O^{*}(n, m)$ and $\delta>0$. 
Proof. It is sufficient to consider $0<\delta<1 / 2$, and we may assume that $p$ is given by

$$
p\left(x_{1}, \ldots, x_{n}\right)=\left(x_{1}, \ldots, x_{m}\right) .
$$

Define $f: B^{m}(0,1 / 2) \times S^{n-m-1} \rightarrow S^{n-1} \subset R^{m} \times R^{n-m}$ by

$$
f(y, z)=\left(y,\left(1-|y|^{2}\right)^{1 / 2} z\right) .
$$

Then $f$ is Lipschitzian and maps $B^{m}(0, \delta) \times S^{n-m-1}$ onto $\left\{x \in S^{n-1}\right.$ : $|p(x)| \leq \delta\}$ for $0<\delta<1 / 2$. Denoting the Lipschitz constant of $f$ by $K$ and using [5, 3.2.23], we find that

$$
\begin{aligned}
& \mathscr{H}^{n-1}\left\{x \in S^{n-1}:|p(x)| \leq \delta\right\} \leq K^{n-1} \mathscr{H}^{n-1}\left[B^{m}(0, \delta) \times S^{n-m-1}\right] \\
= & K^{n-1} \mathscr{L}^{m}\left[B^{m}(0, \delta)\right] \mathscr{H}^{n-m-1}\left(S^{n-m-1}\right)=K^{n-1} \alpha(m) \mathscr{H}^{n-m-1}\left(S^{n-m-1}\right) \delta^{m} .
\end{aligned}
$$

2.5. L e m ma. There is a constant $c_{2}$ depending only on $n$ and $m$ such that

$$
\vartheta_{n, m}^{*}\left\{p \in O^{*}(n, m):|p(x)| \leq \delta\right\} \leq c_{2} \delta^{m}|x|^{-m}
$$

for all $x \in R^{n}$ and $\delta>0$.

Proof. Since $p(x)=|x| p(x /|x|)$ for $x \neq 0$, we may assume that $x \in S^{n-1}$. Fix $q \in O^{*}(n, m)$ and define $\Phi: O(n) \rightarrow S^{n-1}$ by $\Phi(g)=g(x)$ for $g \in O(n)$. Applying statement (3) in [5, 3.2.47] with $r=n-1$, $r+\mu=n(n-1) / 2$ and observing that $\mathscr{H}^{r+\mu}$ is then a constant multiple of $\vartheta_{n}$ because of $[5,3.2 .28(5)]$, we find a constant $c$ depending only on $n$ such that

$$
\vartheta_{n}\left[\Phi^{-1}(E)\right]=c \mathscr{H}^{n-1}(E)
$$

for $E \subset S^{n-1}$. Hence by (2.1) and Lemma 2.4,

$$
\begin{aligned}
& \vartheta_{n, m}^{*}\left\{p \in O^{*}(n, m):|p(x)| \leq \delta\right\} \\
& =\vartheta_{n}\{g \in O(n):|(q \circ g)(x)| \leq \delta\} \\
& =\vartheta_{n}\left(\Phi^{-1}\left\{y \in S^{n-1}:|q(y)| \leq \delta\right\}\right) \\
& =c \mathscr{H}^{n-1}\left\{y \in S^{n-1}:|q(y)| \leq \delta\right\} \leq c c_{1} \delta^{m} .
\end{aligned}
$$

The corresponding inequality for the measure $\gamma_{n, m}$ follows from Lemma 2.5 and (2.3):

2.6. L e m m a. There is a constant $c_{3}$ depending only on $n$ and $m$ such that

$$
\gamma_{n, m}\{V \in G(n, m): \operatorname{dist}(x, V) \leq \delta\} \leq c_{\mathbf{3}} \delta^{n-m}|x|^{m-n}
$$

for all $x \in R^{n}$ and $\delta>0$. 


\section{Integral inequalities}

In this section we assume that

(3.1) $M$ is a separable metric space,

(3.2) $F: R^{n} \times M \rightarrow R^{1}$ is a non-negative Borel function such that $F(x, a) \leq|x|$ for all $(x, a) \in R^{n} \times M$,

(3.3) $\alpha$ and $b$ are positive numbers and $\varphi$ is a Borel regular measure over $M$ such that

$$
\varphi\{a \in M: F(x, a) \leq \delta\} \leq b \delta^{\alpha}|x|^{-\alpha}
$$

for all $x \in R^{n}$ and $\delta>0$.

The separability of $M$ makes the subsequent applications of Fubini's theorem legitimate. The results of this section will be applied with $M=O^{*}(n, m), \quad F:(x, p) \mapsto|p(x)| \quad$ and $\quad M=G(n, m), \quad F:(x, V)$ $\mapsto$ dist $(x, V)$. Lemmas 2.5 and 2.6 guarantee that $\vartheta_{n, m}^{*}$ and $\gamma_{n, m}$ satisfy the condition (3.3) with $\alpha=m$ and $\alpha=n-m$, respectively. In Sections 5 and 6 we shall replace $\varphi$ by other measures over $O^{*}(n, m)$ and $G(n, m)$.

3.4. If $\mu$ measures a set $X$ (i.e. $\mu$ is an (outer) measure over $X$ ), $\mu(X)<\infty, a$ and $t$ are real numbers, $a \geq 0$ and $f: X \rightarrow\{x: 0 \leq$ $x \leq a\}$ is $\mu$ measurable, then

$$
\int_{X} f^{t} d \mu=\int_{0}^{a} r^{t} d \mu\{x \in X: f(x) \leq r\},
$$

where the right hand side is a Riemann-Stieltjes integral. In the cases where $t \geq 0$ or $\inf f(X)>0$, this follows immediately from the fact that $\int_{X} f^{t} d \mu$ lies between the sums $\sum_{i=1}^{k} r_{i}^{t} \mu\left\{x: r_{i-1}<f(x) \leq r_{i}\right\}$ and $\sum_{i=1}^{k} r_{i-1}^{t} \mu\left\{x: r_{i-1}<f(x) \leq r_{i}\right\} \quad$ whenever $0=r_{0}<r_{1}<\ldots<r_{k}=a$. In the general case one can approximate by functions $f_{n}=\sup (f, 1 / n)$, $n=1,2, \ldots$.

3.5. L e m ma. If $0<t<\alpha$ and $x \in R^{n}$, then

$$
\int F(x, a)^{-t} d \varphi a \leq c|x|^{-t}
$$

where $c=b\left[1+t(\alpha-t)^{-1}\right]$.

Proof. Apply 3.4, integrate by parts, and use (3.2) and (3.3) to deduce

$$
\int F(x, a)^{-t} d \varphi a=\int_{0}^{|x|} r^{-t} d \varphi\{a: F(x, a) \leq r\}
$$




$$
\begin{aligned}
& \leq|x|^{-t} \varphi\{a: F(x, a) \leq|x|\}+t \int_{0}^{|x|} r^{-t-1} \varphi\{a: F(x, a) \leq r\} d \mathscr{L}^{1} r \\
& \leq b|x|^{-t}+b t|x|^{-\alpha} \int_{9}^{|x|} r^{\alpha-t-1} d \mathscr{L}^{1} r=b\left[1+t(\alpha-t)^{-1}\right]|x|^{-t} .
\end{aligned}
$$

3.6. L e $\mathrm{m} \mathrm{m}$ a. If $\mu$ is a Borel regular measure over $R^{n}, \mu\left(R^{n}\right)<\infty$, $x \in R^{n}$ and $\delta>0$, then

$$
\int \mu\left\{y \in R^{n}: F(x-y, a) \leq \delta\right\} d \varphi a \leq b \delta^{\alpha} \int|x-y|^{-\alpha} d \mu y .
$$

Proof. Observing that $\left\{(y, a) \in R^{n} \times M: F(x-y, a) \leq \delta\right\}$ is a Borel set and applying Fubini's theorem and (3.3), we obtain

$$
\begin{gathered}
\int \mu\left\{y \in R^{n}: F(x-y, a) \leq \delta\right\} d \varphi a \\
=\int \varphi\{a \in M: F(x-y, a) \leq \delta\} d \mu y \leq b \delta^{\alpha} \int|x-y|^{-\alpha} d \mu y .
\end{gathered}
$$

3.7. If $\mu$ measures $R^{n}, x \in R^{n}, a \in M$ and $r>0$, we define

$$
\begin{aligned}
\psi_{r}^{\alpha}(\mu, x, a)= & \liminf _{\delta \downarrow 0} \delta^{-\alpha} \mu\{y \in B(x, r): F(x-y, a) \leq \delta\}, \\
& \psi^{\alpha}(\mu, x, a)=\lim _{r \downarrow 0} \psi_{r}^{\alpha}(\mu, x, a) .
\end{aligned}
$$

The set $\{(y, x, a): y \in B(x, r), \quad F(x-y, a) \leq \delta\}$ is a Borel set in $R^{n} \times R^{n} \times M$. Therefore $\mu\{y \in B(x, r): F(x-y, a) \leq \delta\}$ is a Borel function with respect to $(x, a)$ according to Fubini's theorem, and it follows by standard methods (cf. $[4,3.4]$ ) that both $\psi_{r}^{\alpha}$ and $\psi^{\alpha}$ are Borel functions with respect to $(x, a)$.

3.8. L e $\mathrm{m} \mathrm{m}$ a. If $\mu$ is a Borel regular measure over $R^{n}, \mu\left(R^{n}\right)<\infty$, $x \in R^{n}$ and $r>0$, then

$$
\int \psi_{r}^{\alpha}(\mu, x, a) d \varphi a \leq b \int_{B(x, r)}|x-y|^{-\alpha} d \mu y .
$$

This follows directly from Fatou's lemma and Lemma 3.6.

\section{Projections into lower dimensional space}

Here we adopt the notation of the previous section with $M=O^{*}(n, m)$, $F:(x, p) \mapsto|p(x)|, \alpha=m$ and $\varphi=\vartheta_{n, m}^{*}$. Then 


$$
\psi_{r}^{m}(\mu, x, p)=\liminf _{\delta \downarrow 0} \delta^{-m} \mu\{y \in B(x, r):|p(x-y)| \leq \delta\} .
$$

For any function $f, N(f, y)$ is the number of points (possibly 0 or $\infty)$ in the set $f^{-1}\{y\}$. If $A$ is a Borel set of $R^{n}$, then the function $y \mapsto N(p \mid A, y)$ is $\mathscr{L}^{m}$ measurable for all $p \in O^{*}(n, m)$ (see $[5,2.10 .10]$ ) and the function $(y, p) \mapsto N(p \mid A, y)$ is $\mathscr{L}^{n} \times \vartheta_{n, m}^{*}$ measurable (see $[5,2.10 .16])$. Actually, it can be seen as in 6.1 that these functions are measurable whenever $A$ is a Suslin set, but we shall not make use of this fact here.

If $\mu$ measures a set $X$ and $A \subset X$, then $\mu \mathrm{L} A$ is the restriction measure defined by $(\mu \mathrm{L} A)(B)=\mu(A \cap B)$ for $B \subset X$.

4.1. L e m m a. If $\mu$ is a Borel regular measure over $R^{n}, E$ is a Borel set of $R^{n}$ with $\mu(E)<\infty, p \in O^{*}(n, m)$ and $h>0$, then

$$
\mu\left\{x \in E: \psi^{m}(\mu \mathrm{L} E, x, p) \leq h\right\} \leq 2^{m} m^{m / 2} h \int N(p \mid E, y) d \mathscr{L}^{m} y .
$$

Proof. It is sufficient to prove that

$$
\mu\left\{x \in E: \psi^{m}(\mu \mathrm{L} E, x, p)<h\right\} \leq 2^{m} m^{m / 2} h \int N(p \mid E, y) d \mathscr{L}^{m} y .
$$

To simplify the notation, we assume that $p$ is given by

$$
p\left(x_{1}, \ldots, x_{n}\right)=\left(x_{1}, \ldots, x_{m}\right) .
$$

Let $r$ and $d$ be positive numbers such that $d<r / n$. We identify $R^{n}=R^{m} \times R^{n-m}$ and express $R^{n-m}$ as

$$
R^{n-m}=\bigcup_{i=1}^{\infty} Q_{i},
$$

where $Q_{i}^{\prime} s$ are closed cubes parallel to the coordinate axis with mutually disjoint interiors and with sidelength $d$. We may assume that $\int N(p \mid E, y) d \mathscr{L}^{m} y<\infty$. Then we can choose the cubes $Q_{i}$ such that $\mathscr{L}^{m}\left(p\left[E \cap\left(R^{m} \times \partial Q_{i}\right)\right]\right)=0$, because $\mathscr{L}^{m}[p(E \cap V)]$ can be positive only for countably many disjoint $(n-1)$-dimensional affine subspaces $V$ of $R^{n}$.

If $x \in R^{n}, \delta>0$ and $\varrho>0$, we denote

$$
\begin{gathered}
I(x, \delta, \varrho)=\left\{y \in R^{n}:\left|x_{i}-y_{i}\right| \leq \delta\right. \\
\left.i=1, \ldots, m,\left|x_{i}-y_{i}\right| \leq \varrho, i=m+1, \ldots, n\right\} .
\end{gathered}
$$

Let

and

$$
f(x)=\liminf _{\delta \downarrow 0} \delta^{-m} \mu[E \cap I(x, \delta, d)] \text { for } x \in R^{n},
$$




$$
E_{i}=E \cap\left\{x \in R^{m} \times Q_{i}: f(x)<h\right\} \text { for } i=1,2, \ldots .
$$

By standard arguments $[4,3.4]$ one can see that $f$ is a Borel function. Let $\varepsilon>0$ and fix $i$. By $[5,2.2 .2]$ there is a closed set $F_{i} \subset E_{i}$ such that

$$
\mu\left(E_{i}\right) \leq(1+\varepsilon) \mu\left(F_{i}\right) .
$$

Next we select closed cubes $P_{j} \subset R^{m}$ parallel to the coordinate axis such that

$$
p\left(F_{i}\right) \subset \bigcup_{j=1}^{\infty} P_{j}, \quad \sum_{j=1}^{\infty} \mathscr{L}^{m}\left(P_{j}\right) \leq \mathscr{L}^{m}\left[p\left(F_{i}\right)\right]+\varepsilon .
$$

Then $F_{i} \subset \cup_{j=1}^{\infty} P_{j} \times Q_{i}$ and

$$
\mu\left(F_{i}\right) \leq \sum_{j=1}^{\infty} \mu\left[F_{i} \cap\left(P_{j} \times Q_{i}\right)\right]
$$

We shall show that

$$
\mu\left[F_{i} \cap\left(P_{j} \times Q_{i}\right)\right] \leq 2^{m} h \mathscr{L}^{m}\left(P_{j}\right) \text { for } j=1,2, \ldots .
$$

Suppose that (1) is false for some $j$. Then dividing $P_{j}$ repeatedly into $2^{m}$ congruent subcubes, we get a decreasing sequence $\left(R_{k}\right)$ of subcubes of $P_{j}$ such that

$$
\begin{gathered}
\operatorname{diam}\left(R_{k}\right)=2^{-k} \operatorname{diam}\left(P_{j}\right), \\
\mu\left[F_{i} \cap\left(R_{k} \times Q_{i}\right)\right]>2^{m} h \mathscr{L}^{m}\left(R_{k}\right) .
\end{gathered}
$$

Since the compact non-empty sets $F_{i} \cap\left(R_{k} \times Q_{i}\right)$ form a decreasing sequence, there is a point $x \in \cap_{k=1}^{\infty} F_{i} \cap\left(R_{k} \times Q_{i}\right)$. Let $\delta_{k}$ be the sidelength of $R_{k}$. Then

$$
F_{i} \cap\left(R_{k} \times Q_{i}\right) \subset E \cap I\left(x, \delta_{k}, d\right),
$$

and it follows from (3) that

$$
\liminf _{k \rightarrow \infty} \delta_{k}^{-m} \mu\left[E \cap I\left(x, \delta_{k}, d\right)\right] \geq 2^{m} h .
$$

For any $0<\delta<\delta_{1}$ there is $k$ such that $\delta_{k} \leq \delta<2 \delta_{k}$ because of (2). Then (4) yields $f(x) \geq h$. But this contradicts with the fact that $x \in E_{i}$. Hence (1) holds, and we get

$$
\begin{aligned}
\mu\left(E_{i}\right) & \leq(1+\varepsilon) \mu\left(F_{i}\right) \leq(1+\varepsilon) \sum_{j=1}^{\infty} \mu\left[F_{i} \cap\left(P_{j} \times Q_{i}\right)\right] \\
& \leq(1+\varepsilon) 2^{m} h \sum_{j=1}^{\infty} \mathscr{L}^{m}\left(P_{j}\right) \leq(1+\varepsilon) 2^{m} h\left(\mathscr{L}^{m}\left[p\left(F_{i}\right)\right]+\varepsilon\right) \\
& \leq(1+\varepsilon) 2^{m} h\left(\mathscr{L}^{m}\left[p\left(E_{i}\right)\right]+\varepsilon\right) .
\end{aligned}
$$


Letting $\varepsilon \downarrow 0$, we have

$$
\mu\left(E_{i}\right) \leq 2^{m} h \mathscr{L}^{m}\left[p\left(E_{i}\right)\right] .
$$

Summing with respect to $i$ and recalling that $\mathscr{L}^{m}\left[p\left(E_{i} \cap E_{i^{\prime}}\right)\right]=0$ for $i \neq i^{\prime}$, we obtain

$$
\begin{gathered}
\mu\{x \in E: f(x)<h\} \leq \sum_{i=1}^{\infty} \mu\left(E_{i}\right) \\
\leq 2^{m} h \sum_{i=1}^{\infty} \mathscr{L}^{m}\left[p\left(E_{i}\right)\right] \leq 2^{m} h \int N(p \mid E, y) d \mathscr{L}^{m} y .
\end{gathered}
$$

Since $d<r / n$, we have

$$
I(x, \delta, d) \subset\left\{y \in B(x, r):|p(x-y)| \leq m^{1 / 2} \delta\right\}
$$

for sufficiently small $\delta>0$. Hence

$$
\mu\left\{x \in E: \psi_{r}^{m}(\mu \mathrm{L} E, x, p)<h\right\} \leq 2^{m} m^{m / 2} h \int N(p \mid E, y) d \mathscr{L}^{m} y .
$$

Letting $r \downarrow 0$ completes the proof.

For the definition of the integralgeometric measure $\mathscr{I}_{1}^{m}$ see $[5,2.10 .5]$.

4.2. Th e o r e m. Let $\mu$ be a Borel regular measure over $R^{n}$ and $E$ a $\mu$ measurable subset of $R^{n}$ with $0<\mu(E)<\infty$. If

$$
\int_{E}|x-y|^{-m} d \mu y<\infty
$$

for $\mu$ almost all $x \in E$, then

$$
\int^{*} N(p \mid E, y) d \mathscr{L}^{m} y=\infty
$$

for $\vartheta_{n, m}^{*}$ almost all $p \in O^{*}(n, m)$, and $\mathscr{I}_{1}^{m}(E)=\infty$.

Proof. Since $E$ contains a closed set of positive $\mu$ measure [5, 2.2.2-3], it is sufficient to prove the theorem in the case where $E$ is closed. Let $P$ be the set of those $p \in O^{*}(n, m)$ for which $\int N(p \mid E, y) d \mathscr{L}^{m} y<\infty$. Then it follows from Lemma 4.1 that $p \in P$ implies $\psi^{m}(\mu \mathrm{L} E, x, p)>0$ for $\mu$ almost all $x \in E$, whence $\int_{E} \psi^{m}(\mu \mathrm{L} E, x, p) d \mu x>0$. On the other hand, under the present assumptions Lemma 3.8 combined with Fatou's lemma shows that $\int \psi^{m}(\mu \mathrm{L} E, x, p) d \vartheta_{n, m}^{*} p=0$ for $\mu$ almost all $x \in E$. Thus by Fubini's theorem,

$\iint_{E} \psi^{m}(\mu \mathrm{L} E, x, p) d \mu x d \vartheta_{n, m}^{*} p=\iint_{E} \psi^{m}(\mu \mathrm{L} E, x, p) d \vartheta_{n, m}^{*} p d \mu x=0$.

Hence $\vartheta_{n, m}^{*}(P)=0$. This proves the first assertion. The second assertion, $\mathscr{I}_{1}^{m}(E)=\infty$, follows from the first and $[5,2.10 .15]$. 
4.3. Capacity. The inner capacity $C_{s}, 0<s<n$, is defined as follows: If $K$ is a compact subset of $R^{n}$, then

$$
C_{s}(K)=\sup \mu\left(R^{n}\right),
$$

where the supremum is taken over all (non-negative) Radon measures $\mu$ such that the support of $\mu$, spt $\mu$, is contained in $K$ and $\int|x-y|^{-s} d \mu y \leq 1$ for all $x \in \operatorname{spt} \mu$. For arbitrary $E \subset R^{n}$, we set

$$
C_{s}(E)=\sup \left\{C_{s}(K): K \subset E, K \text { is compact }\right\} .
$$

The corresponding outer capacity $C_{s}^{*}$ is defined for $E \subset R^{n}$ by

$$
C_{s}^{*}(E)=\inf \left\{C_{s}(G): E \subset G, G \text { is open }\right\} .
$$

Then $C_{s}^{*}(E)=C_{s}(E)$ for all Suslin sets $E$ of $R^{n}$ (see [9, Theorem 2.8, p. 156]).

If $\mu$ is a Radon measure and $\int|x-y|^{-s} d \mu y \leq 1$ for all $x \in \operatorname{spt} \mu$, it follows easily (see [9, Theorem 1.5, p. 66]) that $\int|x-y|^{-s} d \mu y \leq 2^{s}$ for all $x \in R^{n}$. Hence $C_{s}(E)>0$ if and only if there exists a Radon measure $\mu$ such that $\operatorname{spt} \mu \subset E, 0<\mu(E)<\infty$ and $\int|x-y|^{-s} d \mu y \leq 1$ for all $x \in R^{n}$. Thus we obtain from Theorem 4.2:

4.4. The o r em. If $E \subset R^{n}$ is measurable with respect to every Radon measure and if $C_{m}(E)>0$, then $\int^{*} N(p \mid E, y) d \mathscr{L}^{m} y=\infty$ for $\vartheta_{n, m}^{*}$ almost all $p \in O^{*}(n, m)$.

If $E \subset R^{n}$, we denote by $\operatorname{dim} E$ the Hausdorff dimension of $E$ i.e. $\operatorname{dim} E=\inf \left\{s: \mathscr{H}^{s}(E)=0\right\}$. It is well-known that

$$
\operatorname{dim} E=\inf \left\{s: C_{s}^{*}(E)=0\right\}=\sup \left\{s: C_{s}^{*}(E)>0\right\}
$$

(see e.g. [6, Section 2] or [9, Theorem 3.13, p. 196 and Theorem 3.14, p. 200]). Consequently,

$$
\operatorname{dim} E=\inf \left\{s: C_{s}(E)=0\right\}=\sup \left\{s: C_{s}(E)>0\right\},
$$

if $E$ is a Suslin set. Therefore

4.6. Th e o r e m. If $E$ is a Suslin set of $R^{n}$ and $\operatorname{dim} E>m$, then $\int^{*} N(p \mid E, y) d \mathscr{L}^{m} y=\infty$ for $\vartheta_{n, m}^{*}$ almost all $p \in O^{*}(n, m)$.

This theorem could also be deduced directly from Theorem 4.2 , because for $s>m$ the use of Riemann-Stieltjes integration shows that $\int_{E}|x-y|^{-m} d \mathscr{H}^{s} y<\infty \quad$ whenever the upper density $\Theta^{* s}\left(\mathscr{H}^{s} \mathrm{~L} E, x\right)$ is finite.

In case $E$ is a Borel set, the conclusion in 4.4 and 4.6 implies that $\mathscr{I}_{1}^{m}(E)=\infty$. But since $\mathscr{I}_{1}^{m}$ is Borel regular $[5,2.10 .1]$, we have more generally

4.7. Theorem. If $E \subset R^{n}$ and $\mathscr{I}_{1}^{m}(E)<\infty$, then $C_{m}(E)=0$ and $\operatorname{dim} E \leq m$. 


\section{Projections into higher dimensional space}

In the first three lemmas of this section, we assume that $0<\alpha \leq m$, $b>0$ and $\varphi$ is a Borel regular measure over $O^{*}(n, m)$ such that

$$
\varphi\left\{p \in O^{*}(n, m):|p(x)| \leq \delta\right\} \leq b \delta^{\alpha}|x|^{-\alpha}
$$

for all $x \in R^{n}$ and $\delta>0$.

If $0<s<m$ and $\mu$ is a Radon measure over $R^{n}$, we denote the $s$-energy of $\mu$ by $I_{s}(\mu)$ i.e.

$$
I_{s}(\mu)=\iint|x-y|^{-s} d \mu y d \mu x
$$

Then for compact sets $K \subset R^{n}$,

$$
C_{s}(K)^{-1}=\inf I_{s}(\mu),
$$

where the infimum is taken over all Radon measures $\mu$ such that spt $\mu \subset K$ and $\mu(K)=1$ (see [9, p. 139]).

5.1. L e m m a. If $0<s<\alpha$ and $K$ is a compact set of $R^{n}$, then

$$
\int C_{s}[p(K)]^{-1} d \varphi p \leq c C_{s}(K)^{-1}
$$

where $c=b\left[1+s(\alpha-s)^{-1}\right]$.

Proof. The integrand is measurable; in fact, the function $p \mapsto C_{s}[p(K)]$ is upper semicontinuous in $O^{*}(n, m)$. To see this, let $p \in O^{*}(n, m)$ and $\varepsilon>0$. Then there is an open set $G \supset p(K)$ such that $C_{s}(G) \leq$ $C_{s}[p(K)]+\varepsilon$. When $q$ is sufficiently close to $p$, then $q(K) \subset G$, whence $C_{s}[q(K)] \leq C_{s}(G) \leq C_{s}[p(K)]+\varepsilon$.

Let $\mu$ be a Radon measure such that $\operatorname{spt} \mu \subset K$ and $\mu(K)=1$. If $p \in O^{*}(n, m)$, then the measure $p_{\#} \mu$, defined by $p_{\#} \mu(A)=$ $\mu\left[p^{-1}(A)\right]$ for $A \subset R^{m}$, is a Radon measure over $R^{m}[5,2.2 .17]$, spt $p_{\#} \mu \subset p(K)$ and $p_{\#} \mu[p(K)]=1$. By $[5,2.4 .18(1)]$, we have

$$
I_{s}\left(p_{\#} \mu\right)=\iint|p(x-y)|^{-s} d \mu y d \mu x .
$$

Fubini's theorem and Lemma 3.5 yield

$$
\int I_{s}\left(p_{\#} \mu\right) d \varphi p=\iiint|p(x-y)|^{-s} d \varphi p d \mu y d \mu x \leq c I_{s}(\mu),
$$

from which the lemma follows.

5.2. L e m m a. If $0<s<\alpha, E \subset R^{n}$ and $C_{s}(E)>0$, then there is a Borel set $P \subset O^{*}(n, m)$, independent of $\varphi$, such that $\varphi(P)=0$ and $C_{s}[p(E)]>0$ for all $p \in O^{*}(n, m) \backslash P$. 
Proof. Choose a compact set $K \subset E$ such that $C_{s}(K)>0$. Then the set

$$
P=\left\{p \in O^{*}(n, m): C_{s}[p(K)]=0\right\}
$$

has the required properties by Lemma 5.1 and its proof.

5.3. L e m ma. If $E$ is a Suslin set of $R^{n}$ and $\operatorname{dim} E \leq \alpha$, then there is a Borel set $P \subset O^{*}(n, m)$, independent of $\varphi$, such that $\varphi(P)=0$ and $\operatorname{dim} p(E)=\operatorname{dim} E$ for all $p \in O^{*}(n, m) \backslash P$.

This follows from (4.5) and 5.2 if we choose $P=\cup_{j=1}^{\infty} P_{j}$, where $P_{j}$ is the Borel set of Lemma 5.2 corresponding to $s=\operatorname{dim} E-1 / j$.

By Lemma 2.5, the above results hold with $\alpha=m$ and $\varphi=\vartheta_{n, m}^{*}$. We now proceed to obtain more exact information about the Hausdorff measures of the exceptional sets of Lemmas 5.2 and 5.3. Recall from [5, 3.2.28] that $O^{*}(n, m)$ is an $m(2 n-m-1) / 2$ dimensional submanifold of class $\infty$ of the euclidean space $\operatorname{Hom}\left(R^{n}, R^{m}\right)$ with

$$
0<\mathscr{H}^{m(2 n-m-1) / 2}\left[O^{*}(n, m)\right]<\infty .
$$

We use the norm $\|f\|=\sup \{|f(x)|:|x|=1\}$ on $\operatorname{Hom}\left(R^{n}, R^{m}\right)$. Then $B(f, r)=\left\{g \in \operatorname{Hom}\left(R^{n}, R^{m}\right):\|f-g\| \leq r\right\}$ for $f \in \operatorname{Hom}\left(R^{n}, R^{m}\right)$ and $r>0$.

We shall apply the following theorem, which is due to $O$. Frostman. A proof is given in [2, pp. $7-8]$.

5.4. Th e o r e m. Let $E$ be a Sustin set of $R^{n}$ and $s \geq 0$. Then $\mathscr{H}^{s}(E)>0$ if and only if there exists a Borel regular measure $\varphi$ such that $0<\varphi(E)<\infty$ and $\varphi[B(x, r)] \leq r^{s}$ for all $x \in R^{n}$ and $r>0$.

To be able to combine the above theorem with the lemmas of this section, we prove the following

5.5. $\mathrm{L}$ e $\mathrm{m} \mathrm{m}$ a. If $\varphi$ is a Borel regular measure over $O^{*}(n, m)$ with $\varphi\left[O^{*}(n, m)\right]<\infty, \quad t \geq \boldsymbol{v}=m(2 n-m-3) / 2$ and

$$
\varphi\left[O^{*}(n, m) \cap B(p, \delta)\right] \leq \delta^{t}
$$

for all $p \in O^{*}(n, m)$ and $\delta>0$, then

$$
\varphi\left\{p \in O^{*}(n, m):|p(x)| \leq \delta\right\} \leq b \delta^{t-v}|x|^{\nu-t}
$$

for all $x \in R^{n}$ and $\delta>0$, where $b$ is a constant depending only on $n$, $m$ and $t$.

Proof. We may assume that $x \in S^{n-1}$ and $\delta<1$. Put

$$
\begin{aligned}
A_{\delta} & =\left\{p \in O^{*}(n, m):|p(x)| \leq \delta\right\}, \\
B & =\left\{p \in O^{*}(n, m): p(x)=0\right\} .
\end{aligned}
$$

Let $p \in A_{\delta}$. Then there is $a \in S^{n-1} \cap \operatorname{ker} p$ such that $|x-a| \leq 2 \delta$. Choose $g \in O(n)$ so that $g(x)=a$ and $\left\|g-\mathbf{1}_{R^{n}}\right\|=|x-a|$. One can 
find such a $g$ by writing $R^{n}=V \oplus V^{\perp}$, where $\{x, a\} \subset V \in G(n, 2)$ and $V^{\perp}$ is the orthogonal complement of $V$, letting $f$ be a rotation in $V$ for which $f(x)=a$ and taking $g=f \oplus \mathbf{1}_{V^{\perp}}$. Then $\|p-p \circ g\| \leq 2 \delta$ and $p \circ g \in B$. This shows that

$$
A_{\delta} \subset\left\{p \in O^{*}(n, m): \operatorname{dist}(p, B) \leq 2 \delta\right\} .
$$

For $p \in O^{*}(n, m)$, we denote by $p^{*}$ the adjoint of $p[5,1.7 .4]$ and let $O(n, m)=\left\{p^{*}: p \in O^{*}(n, m)\right\}$ be the set of all orthogonal injections $R^{m} \rightarrow R^{n}$. If $p \in B$, the image im $p^{*}$ is contained in $\left\{y \in R^{n}: y \cdot x=0\right\}$. Choosing a linear isometry $f:\left\{y \in R^{n}: y \cdot x=\right.$ $0\} \rightarrow R^{n-1}$, we can define an isometry $F: B \rightarrow O(n-1, m)$ by $F(p)=$ $f \circ p^{*}$. Thus it follows from $[5,3.2 .28(5)]$ that $0<\mathscr{H}^{v}(B)<\infty$ and that $B$ is a $\nu$-dimensional manifold of class $\infty$. Hence the upper density $\Theta^{*^{v}}\left(\mathscr{H}^{v} \mathrm{~L} B, p\right)$ is positive for all $p \in B$. Moreover, $\mathscr{H}^{v}[B \cap B(p, r)]$ is independent of $p$ for all $r>0$. Consequently, there is a positive number $c$ depending only on $n$ and $m$ such that

$$
\mathscr{H}^{v}[B \cap B(p, r)] \geq c r^{v}
$$

for all $p \in B$ and $0<r<1$. From (1) it follows that

$$
\mathscr{H}^{v}[B \cap B(p, 3 \delta)] \geq c \delta^{v}
$$

for $p \in A_{\delta}$. We define a Borel function $f: A_{\delta} \times B \rightarrow R^{1}$ setting $f(p, q)=1$, if $p \in B(q, 3 \delta)$, and $f(p, q)=0$ otherwise. Then by Fubini's theorem,

$$
\begin{gathered}
c \delta^{v} \varphi\left(A_{\delta}\right) \leq \int_{A_{\delta}} \mathscr{H}^{v}[B \cap B(p, 3 \delta)] d \varphi p=\iint_{A_{\delta}} f(p, q) d \mathscr{H}^{v} q d \varphi p \\
=\int_{B} \int_{A_{\delta}} f(p, q) d \varphi p d \mathscr{H}^{v} q=\int_{B} \varphi\left[A_{\delta} \cap B(q, 3 \delta)\right] d \mathscr{H}^{v} q \leq 3^{t} \delta^{t} \mathscr{H}^{v}(B),
\end{gathered}
$$

and $\varphi\left(A_{\delta}\right) \leq b \delta^{t-v}$ with $b=3^{t} c^{-1} \mathscr{H}^{v}(B)$. This completes the proof.

Combining $5.2-5.5$, we can state the results of this section as follows:

5.6. Theorem. Let $E \subset R^{n}$.

(a) If $0<s<m$ and $C_{s}(E)>0$, then $C_{s}[p(E)]>0$ for $\vartheta_{n, m}^{*}$ almost all $p \in O^{*}(n, m)$. More precisely, the Hausdorff dimension of the exceptional set is at most $s+m(2 n-m-3) / 2$.

(b) If $E$ is a Suslin set and $s=\operatorname{dim} E \leq m$, then $\operatorname{dim} p(E)=\operatorname{dim} E$ for $\vartheta_{n, m}^{*}$, more precisely $\mathscr{H}^{s+m(2 n-m-3) / 2}$, almost all $p \in O^{*}(n, m)$.

5.7. Remarks. (1) If $V \in G(n, m)$, let $P_{V}$ be the orthogonal projection from $R^{n}$ onto $V$. By similar methods as above, one can prove that

$$
\operatorname{dim}\left\{V \in G(n, m): C_{s}\left[P_{V}(E)\right]=0\right\} \leq s+m(n-m-1)
$$


with the assumptions of $5.6(\mathrm{a})$, and

$$
\mathscr{H}^{s+m(n-m-1)}\left\{V \in G(n, m): \operatorname{dim} P_{V}(E)<\operatorname{dim} E\right\}=0
$$

with the assumptions of $5.6(\mathrm{~b})$.

(2) In [8] Kaufman has given an example of a compact plane set $D$ such that $\operatorname{dim} D \leq 1$ and that the set of those $p \in O^{*}(2,1)$ for which $\operatorname{dim} p(D)<\operatorname{dim} D$ is of positive Hausdorff dimension. Later it has been shown (unpublished) first by Kaufman in the case $n=2$ and then by Kaufman and the present author in co-operation in the general case that $s+m(2 n-m-3) / 2$ is the best possible upper bound for the Hausdorff dimension of the exceptional set of Theorem 5.6.

(3) It follows from [9, Theorem 2.9, p. 158] that $C_{s}^{*}[p(E)] \leq C_{s}^{*}(E)$ for all $E \subset R^{n}$ and $p \in O^{*}(n, m)$.

\section{Intersections with planes}

Here we use the methods of Marstrand [10, Section 6] to examine the intersections of an $s$-dimensional set with $m$-dimensional affine subspaces of $R^{n}$. If $x \in R^{n}$ and $A \subset R^{n}$, we denote by $\tau_{x} A$ the $x$-translate $\left\{y \in R^{n}: y-x \in A\right\}$ of $A$.

6.1. Lemma. If $E$ is a Suslin set of $R^{n}$ and $t \geq 0$, then the function

$$
(x, V) \mapsto \mathscr{H}^{t}\left(E \cap \tau_{x} V\right)
$$

is a Suslin function in $R^{n} \times G(n, m)$, i.e. the inverse image of $\{v: v>u\}$ is a Suslin set in $R^{n} \times G(n, m)$ for all $u \geq 0$.

Proof. We shall apply the following statement, which is a special case of a result of Dellacherie [3, Chapitre VI, Corollaire 21]:

If $A$ is a Suslin set in $R^{n} \times R^{n} \times G(n, m)$ and $A(x, V)=$ $\left\{y \in R^{n}:(y, x, V) \in A\right\}$ for $(x, V) \in R^{n} \times G(n, m)$, then the function $(x, V) \mapsto \mathscr{H}^{t}[A(x, V)]$ is a Suslin function in $R^{n} \times G(n, m)$.

We choose

$$
\begin{aligned}
A & =\left\{(y, x, V): y \in E \cap \tau_{x} V\right\} \\
& =\left[E \times R^{n} \times G(n, m)\right] \cap\{(y, x, V): x-y \in V\} .
\end{aligned}
$$

Then $A$ is a Suslin set as an intersection of a Suslin set and a closed set, $A(x, V)=E \cap \tau_{x} V$, and the lemma follows.

We adopt the notation of Section 3 with $M=G(n, m)$ and $F(x, V)=$ dist $(x, V)$ for $(x, V) \in R^{n} \times G(n, m)$. Then if $\mu$ measures $R^{n}, x \in R^{n}$, $V \in G(n, m)$ and $r>0$, we have 


$$
\psi_{r}^{n-m}(\mu, x, V)=\liminf _{\delta \downarrow 0} \delta^{m-n} \mu\{y \in B(x, r): \text { dist }(x-y, V) \leq \delta\} .
$$

The following lemma can be proved by the same method as Lemma 16 in $[10]$. We omit the details.

6.2. L e m m a. Suppose that $E$ is an $\mathscr{H}^{s}$ measurable subset of $R^{n}$ with $0<\mathscr{H}^{s}(E)<\infty, t>0$ and $V \in G(n, m)$. If $\mathscr{H}^{t}\left(E \cap \tau_{a} V\right)=$ 0 for all $a \in R^{n}$, then

$$
\limsup _{r \downarrow 0} r^{-t} \psi_{r}^{n-m}\left(\mathscr{H}^{s} \mathrm{~L} E, x, V\right)=\infty
$$

for $\mathscr{H}^{\text {s }}$ almost all $x \in E$.

6.3. L e m ma. If $0<u<s, E \subset R^{n}$ and $\mathscr{H}^{s}(E)<\infty$, then

$$
\limsup _{r \downarrow 0} r^{u-s} \int_{E \cap B(x, r)}|x-y|^{-u} d \mathscr{H}^{s} y \leq s(s-u)^{-1}
$$

for $\mathscr{H}^{s}$ almost all $x \in R^{n}$.

Proof. Let $x \in R^{n}$ and $r>0$. Applying 3.4 and integrating by parts, we have

$$
\begin{gathered}
\int_{E \cap B(x, r)}|x-y|^{-u} d \mathscr{H}^{s} y=\int_{0}^{r} \varrho^{-u} d \mathscr{H}^{s}[E \cap B(x, \varrho)] \\
\leq r^{-u} \mathscr{H}^{s}[E \cap B(x, r)]+u \int_{0}^{r} \varrho^{-u-1} \mathscr{H}^{s}[E \cap B(x, \varrho)] d \mathscr{L}^{1} \varrho .
\end{gathered}
$$

The assertion follows from the fact that the upper density $\Theta^{* s}\left(\mathscr{H}^{s} L E, x\right)$ is at most one for $\mathscr{H}^{s}$ almost all $x \in R^{n}[5,2.10 .19(5)]$.

6.4. L e m ma. If $E$ is an $\mathscr{H}^{s}$ measurable subset of $R^{n}$ with $0<\mathscr{H}^{s}(E)<\infty$, then

$$
\operatorname{dim} E \cap \tau_{x} V \geq s+m-n
$$

for $\mathscr{H}^{s} \times \gamma_{n, m}$ almost all $(x, V) \in E \times G(n, m)$.

Proof. It is sufficient to prove this for an arbitrary compact subset $F$ of $E$ and $s+m-n>0$. Let $0<t<s+m-n$. Denoting

$$
f(x, V)=\lim _{r \downarrow 0} \sup ^{-t} \psi_{r}^{n-m}\left(\mathscr{H}^{s} \mathrm{~L} F, x, V\right)
$$

for $(x, V) \in R^{n} \times G(n, m)$, we infer with the help of Lemmas $3.8,6.3$ and the obvious generalization of $\left[10\right.$, Lemma 10] that $\int f(x, V) d \gamma_{n, m} V=0$ for $\mathscr{H}^{s}$ almost all $x \in F$, whence

$$
\iint_{F} f d \mathscr{H}^{s} d \gamma_{n, m}=0 .
$$


Applying Lemma 6.2 to the set

$$
E_{V}=\left\{x \in F: \mathscr{H}^{t}\left(F \cap \tau_{x} V\right)=0\right\}
$$

for $V \in G(n, m)$, we find that $f(x, V)=\infty$ for $\mathscr{H}^{s}$ almost all $x \in E_{V}$. Hence (1) implies that $\mathscr{H}^{s}\left(E_{V}\right)=0$ for $\gamma_{n, m}$ almost all $V \in G(n, m)$. The set $\left\{(x, V): x \in E_{V}\right\}$ is $\mathscr{H}^{s} \times \gamma_{n, m}$ measurable by Lemma 6.1, and Fubini's theorem can be used to show that $\mathscr{H}^{s} \times \gamma_{n, m}\left\{(x, V): x \in E_{V}\right\}=0$. The lemma follows by the arbitrariness of $t$.

6.5. L e m ma. Suppose that $r$ and $s$ are positive numbers, $x \in R^{n}$, $E \subset R^{n}$ and $\mathscr{H}^{s}(E)<\infty$. If $s \geq n-m$, then

$$
\int^{*} \mathscr{H}^{s+m-n}\left([E \backslash B(x, r)] \cap \tau_{x} V\right) d \gamma_{n, m} V \leq c r^{m-n} \mathscr{H}^{s}[E \backslash B(x, r)],
$$

where $c$ is a constant depending only on $n, m$ and $s$. If $s<n-m$, then

$$
[E \backslash B(x, r)] \cap \tau_{x} V=\varnothing
$$

for $\gamma_{n, m}$ almost all $V \in G(n, m)$.

Proof. For every positive integer $k$, choose balls $B_{k, i} \subset R^{n}$, $i=1,2, \ldots$, such that

$$
\begin{gathered}
E \backslash B(x, r) \subset \bigcup_{i=1}^{\infty} B_{k, i} \subset R^{n} \backslash B(x, r / 2), \\
\sum_{i=1}^{\infty} \alpha(s) 4^{-s}\left(\operatorname{diam} B_{k, i}\right)^{s}<\mathscr{H}^{s}[E \backslash B(x, r)]+k^{-1}, \\
\operatorname{diam} B_{k, i}<k^{-1}, \quad i=1,2, \ldots
\end{gathered}
$$

Since $B_{k, i} \subset R^{n} \backslash B(x, r / 2)$, we obtain from Lemma 2.6 that

$$
\begin{aligned}
& \gamma_{n, m}\left\{V \in G(n, m): B_{k, i} \cap \tau_{x} V \neq \varnothing\right\} \leq c_{3} 2^{n-m} r^{m-n}\left(\operatorname{diam} B_{k, i}\right)^{n-m} . \\
& \text { If } s \geq n-m \text {, we get } \\
& \qquad\left[\operatorname{diam}\left(B_{k, i} \cap \tau_{x} V\right)\right]^{s+m-n} d \gamma_{n, m} V \leq c_{3} 2^{n-m} r^{m-n}\left(\operatorname{diam} B_{k, i}\right)^{s} .
\end{aligned}
$$

Here we agree that $\operatorname{diam} \varnothing=0$. The integrand is continuous, and therefore measurable. We sum over $i$ and use Fatou's lemma to infer

$$
\begin{aligned}
& \int \liminf _{k \rightarrow \infty} \sum_{i=1}^{\infty}\left[\operatorname{diam}\left(B_{k, i} \cap \tau_{x} V\right)\right]^{s+m-n} d \gamma_{n, m} V \\
& \leq \alpha(s)^{-1} 4^{s} c_{3} 2^{n-m} r^{m-n} \mathscr{H}^{s}[E \backslash B(x, r)] .
\end{aligned}
$$

But 


$$
\begin{gathered}
\mathscr{H}^{s+m-n}\left([E \backslash B(x, r)] \cap \tau_{x} V\right) \\
\leq \liminf _{k \rightarrow \infty} \sum_{i=1}^{\infty} \alpha(s+m-n) 2^{n-m-s}\left[\operatorname{diam}\left(B_{k, i} \cap \tau_{x} V\right)\right]^{s+m-n},
\end{gathered}
$$

and the first assertion follows.

If $s<n-m$, we conclude for all $k=1,2, \ldots$ that

$$
\begin{aligned}
& \gamma_{n, m}\left\{V \in G(n, m):[E \backslash B(x, r)] \cap \tau_{x} V \neq \varnothing\right\} \\
& \leq \sum_{i=1}^{\infty} \gamma_{n, m}\left\{V \in G(n, m): B_{k, i} \cap \tau_{x} V \neq \varnothing\right\} \\
& \leq c_{3} 2^{n-m} r^{m-n} \sum_{i=1}^{\infty}\left(\operatorname{diam} B_{k, i}\right)^{n-m} \\
& \leq \alpha(s)^{-1} 4^{s} c_{3} 2^{n-m} r^{m-n} k^{m+s-n}\left(\mathscr{H}^{s}[E \backslash B(x, r)]+k^{-1}\right) .
\end{aligned}
$$

Letting $k \rightarrow \infty$ gives the second assertion.

6.6. The or e m. If $s>n-m$ and $E$ is a Suslin set of $R^{n}$ with $0<\mathscr{H}^{s}(E)<\infty$, then

$$
\operatorname{dim} E \cap \tau_{x} V=s+m-n
$$

and

$$
\mathscr{H}^{s+m-n}\left(E \cap \tau_{x} V\right)<\infty
$$

for $\mathscr{H}^{s} \times \gamma_{n, m}$ almost all $(x, V) \in E \times G(n, m)$.

Proof. Use Riemann-Stieltjes integration to deduce from Lemma 6.5 (as in [10, Lemma 19]) that

$$
\begin{gathered}
\int \mathscr{H}^{*+m-n}\left(E \cap \tau_{x} V\right) d \gamma_{n, m} V \\
\leq c r^{m-n} \mathscr{H}^{s}(E)+c \int_{0}^{r} \varrho^{m-n-1} \mathscr{H}^{s}[E \cap B(x, \varrho)] d \mathscr{L}^{1} \varrho
\end{gathered}
$$

for $x \in R^{n}$ and $r>0$. The right hand side is finite, whenever the upper density $\Theta^{* s}\left(\mathscr{H}^{s} \mathrm{~L} E, x\right)$ is finite. This happens for $\mathscr{H}^{s}$ almost all $x \in E[5,2.10 .19(5)]$, whence

$$
\gamma_{n, m}\left\{V \in G(n, m): \mathscr{H}^{s+m-n}\left(E \cap \tau_{x} V\right)=\infty\right\}=0
$$

for $\mathscr{H}^{s}$ almost all $x \in E$. The assertions follow now from Lemmas 6.4, 6.1 and Fubini's theorem.

6.7. Remark. It seems to be an open question whether Theorem 6.6 holds for $s=n-m$. See remarks in [10, 7.3 and 8.1] and [5. 3.3.11].

6.8. The or em. If $s<n-m, x \in R^{n}, E \subset R^{n}$ and $\mathscr{H}^{s}(E)<\infty$, then 


$$
(E \backslash\{x\}) \cap \tau_{x} V=\varnothing
$$

for $\gamma_{n, m}$ almost all $V \in G(n, m)$.

This follows directly from Lemma 6.5.

In the last two theorems, we give some information about the Hausdorff measures of exceptional sets. To compare with Theorems 6.6 and 6.8 , note that $\operatorname{dim} G(n, m)=m(n-m)=n-m+(m-1)(n-m)$.

6.9. The o r e m. If $0<t<s$ and $E$ is a Suslin set of $R^{n}$ with $\mathscr{H}^{s}(E)<\infty$, then

$$
\mathscr{H}^{t}\left(E \cap \tau_{x} V\right)<\infty
$$

for $\mathscr{H}^{s} \times \mathscr{H}^{\alpha}$ almost all $(x, V) \in E \times G(n, m)$, where

$$
\alpha=s-t+(m-1)(n-m) \text {. }
$$

6.10. Th e o r e m. If $s>0, x \in R^{n}$ and $E$ is a Suslin set of $R^{n}$ with $\mathscr{H}^{s}(E)<\infty$, then

$$
\operatorname{dim}\left\{V \in G(n, m):(E \backslash\{x\}) \cap \tau_{x} V \neq \varnothing\right\} \leq s+(m-1)(n-m) .
$$

Proofs. When $G(n, m)$ is identified with a subset of $\bigodot_{2} \wedge_{m} R^{n}$ as in $[5,3.2 .28(4)]$, the norm || on $\bigodot_{2} \wedge_{m} R^{n} \quad[5,1.10 .5]$ induces a metric $d$ on $G(n, m)$. Let $\beta>v=(m-1)(n-m)$ and let $\varphi$ be a Borel regular measure over $G(n, m)$ such that $\varphi[B(V, \delta)] \leq \delta^{\beta}$ for all $V \in G(n, m)$ and $\delta>0$. Then a proof similar to that of Lemma 5.5 shows that there is a constant $b$ depending only on $n, m$ and $\beta$ such that

$$
\varphi\{V \in G(n, m): \operatorname{dist}(x, V) \leq \delta\} \leq b \delta^{\beta-\nu}|x|^{\nu-\beta}
$$

for all $x \in R^{n}$ and $\delta>0$. In fact, assume that $x \in S^{n-1}, \delta<1$, and denote

$$
\begin{gathered}
A_{\delta}=\{V \in G(n, m): \operatorname{dist}(x, V) \leq \delta\}, \\
B=\{V \in G(n, m): x \in V\} .
\end{gathered}
$$

Let $V \in A_{\delta}$ and choose $g \in O(n)$ such that $g(V) \in B$ and $\left\|g-\mathbf{1}_{R^{n}}\right\| \leq$ $2 \delta$. If $V$ is associated with a simple unit $m$-vector $\zeta[5,1.6 .1]$, then $g(V)$ is associated with $\wedge_{m} g(\zeta)$. Using [5, 3.2.28(4) and 1.7.5], one finds that $d[V, g(V)] \leq\left|\zeta-\wedge_{m} g(\zeta)\right| \leq 2 m \delta$. Hence

$$
A_{\delta} \subset\{V: \operatorname{dist}(V, B) \leq 2 m \delta\} \text {. }
$$

Moreover, $B$ is isometric with $G(n-1, m-1)$. The rest of the argument reads as in 5.5.

The proof of Lemma 6.5 now holds with $\gamma_{n, m}$ replaced by $\varphi$ and $n-m$ replaced by $\beta-v$. The ecxeptional sets in Theorems 6.9 and 6.10 are Suslin sets by Lemma 6.1. One can complete the proof of Theorem 6.9 
choosing $\beta=\alpha$, arguing as in 6.6 and using Theorem 5.4. Theorem 6.10 follows with $\beta>s+v$ arbitrary, and with the help of the above mentioned generalization of Lemma 6.5 and Theorem 5.4.

\section{References}

[1] Besicovitch, A. S.: Concentrated and rarified sets of points. - Acta Math. $62,1933,289-300$.

[2] Carlesson, L.: Selected Problems on Exceptional Sets. - Van Nostrand, Princeton, 1968.

[3] Deiracherie, C.: Ensembles Analytiques, Capacités, Mesures de Hausdorff. Springer-Verlag, Berlin-Heidelberg-New York, 1972.

[4] Federer, H.: The $(\phi, k)$ rectifiable subsets of $n$ space. - Trans. Amer. Math. Soc. $62,1947,114-192$.

[5] - - Geometric Measure Theory. - Springer-Verlag, Berlin-HeidelbergNew York, 1969.

[6] -"- Slices and potentials. - Indiana Univ. Math. J. 21, 1971, 373-382.

[7] Kaufman, R.: On Hausdorff dimension of projections. - Mathematika 15, $1968,153-155$.

[8] - - An exceptional set for Hausdorff dimension. - Mathematika 16, 1969, $57-58$.

[9] Landkof, N. S.: Foundations of Modern Potential Theory. - Springer-Verlag, Berlin-Heidelberg-New York, 1972.

[10] Marstrand, J. M.: Some fundamental geometrical properties of plane sets of fractional dimensions. - Proc. London Math. Soc. (3) 4, 1954, 257-302.

University of Helsinki

Department of Mathematics

SF-00100 Helsinki 10

Finland

Received 19 March 1975 